\title{
Is there Weakness Area in Airway?
}

Hirai $S^{1}$, Ando $E^{1}$, Shigeta $Y^{1^{*}}$, Ishikawa $C^{1}$, Ito $T^{1}$, Shigemoto $S^{1}$, Ogawa $T^{1}$, Clark GT $^{2}$ and Enciso $R^{3}$ ${ }^{1}$ Department of Fixed Prosthodontics, School of Dental Medicine, Tsurumi University, Yokohama, J apan ${ }^{2}$ Orofacial Pain/ Oral Medicine Center, Division of Diagnostic Sciences, Ostrow School of Dentistry, University of Southern California, Los Angeles, USA ${ }^{3}$ Division of Craniofacial Sciences and Therapeutics, Ostrow School of Dentistry, University of Southern California, Los Angeles, USA

*Corresponding author: Shigeta Y, Department of Fixed Prosthodontics, School of Dental Medicine, Tsurumi University, Tsurumi-ku, Yokohama, J apan

Received: December 14, 2018; Accepted: January 08, 2019; Published: January 15, 2019

\section{Abstract}

Purpose: In our previous study, it was reported that the cross-section airway of OSA presented triangle shape, especially in the obese patients. From this finding, we hypothesized that there is structural weakness of airway in the bilateral posterior region. In this short report, to discover the weakness region in the airway, the airway configuration change under the negative pressure loading was observed.

Methods: Subject was 33 year-old female, who had no OSA. It was investigated the pressure level of which she was able to keep same the same level stably and constantly for 15 seconds using a pressure gauge. This pressure level was applied during scanning CBCT (S_NP). The airway was scanned via a CBCT with 15 seconds, under following conditions; normal breathing and under S_NP. The airway volumes between the level of the posterior nasal spine and the tip of epiglottis were measured.

Results: The airway configuration during normal breathing was elliptical shape. On the other hand, the airway under S_NP presented triangle shape. The airway volumes were $16040 \mathrm{~mm}^{3}$ during normal breathing, and $6567 \mathrm{~mm}^{3}$ under S_NP. The airway volume under S_NP decreased to $41 \%$, compared with normal breathing.

Conclusion: In this short report, it could observe the airway configuration under the negative pressure. As a result, the airway may be obstructed from posterolateral direction by fat tissue or negative pressure. This short report provided an importance of research regarding to the structural weakness in the airway.

Keywords: OSA; Negative Pressure Loading; Airway Configuration; CBCT

\section{Introduction}

Obstructive Sleep Apnea (OSA) is characterized by recurrent collapse of the pharyngeal airway during sleep.

In 2008, we have reported the relationship between Body mass index (BMI) and airway configuration. Maximum anterior-posterior diameter (AP) and lateral width (LW) of the cross-section airway were measured, and the square area (SA) was calculated. The airway cross-section area (AWA) was also measured, and then the AWA/SA ratio was calculated. The mean AWA/SA score was $11.2 \%$ smaller in OSA patients and this difference was statistically significant [1]. From this result, the difference of airway configuration between non-OSA and OSA patients was quantitatively revealed.

In the qualitative observation, the airway of non-OSA was square or elliptical shape. On the other hand, the airway of OSA presented triangle shape, especially in the obese patients (Figure 1).

From this finding, we hypothesized that there is structural weakness of airway in the bilateral posterior region.

In this short report, to discover the weakness region in the airway, the airway configuration change under negative pressure was observed.

\section{Methods}

\section{Subject}

Subject was 33 year-old, female, who had no OSA. She agreed with the purpose of this study, and signed. Her body mass index was $19 \mathrm{~kg} / \mathrm{m}^{2}$.

This research was approved by the Ethical Committee at Tsurumi University Dental Hospital (Approval No. 1403).

\section{Measurement of maximum negative pressure (Max.NP)}

Figure 2 showed the overview of our setting. The respiratory mask and the syringe were connected, and the pressure gage (APV40, Keyence Japan, Osaka) was set on the syringe (Figure 2 upper).

The mask was pushed onto her face, and covered her nose and mouth. She performed her maximal inspiration, and the negative pressure within the syringe was measured as Max.NP.

Subsequently, it was investigated the pressure level that she was able to keep the same level of pressure stably and constantly for 15 seconds. This pressure level was applied during CBCT scanning ( $\mathrm{S}_{-}$ $\mathrm{NP})$.

\section{Evaluation of airway configuration and volume}

The airway was scanned via a CBCT (GALILEOS, Sirona, USA, NY) with 15 seconds, under following conditions; normal breathing and under S_NP (Figure 2 lower). 

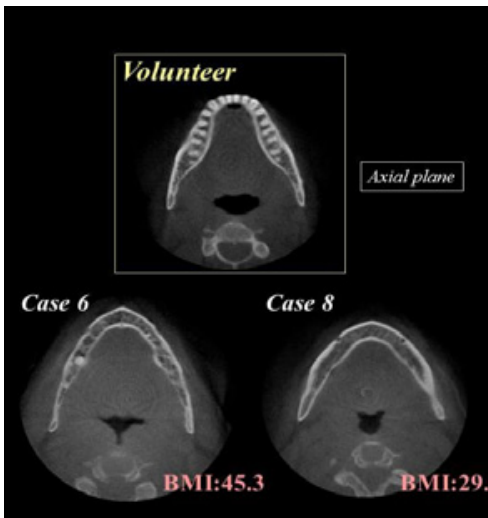

Case 8

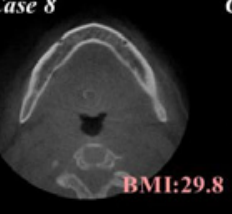

ase 9

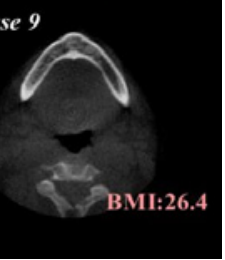

Figure 1: Airway in non-OSA and OSA patients.
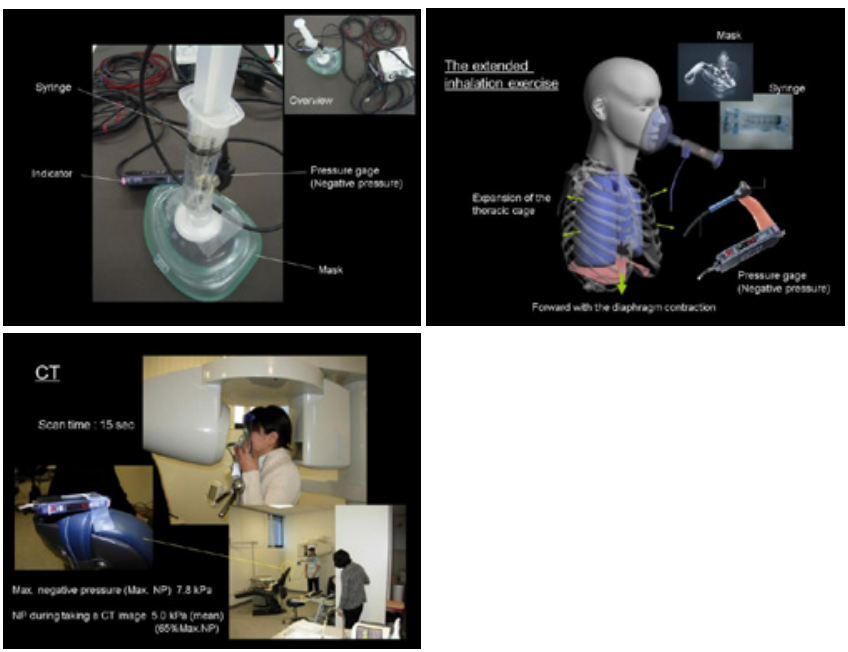

Figure 2: Measurement device for the negative pressure (upper) and overview of our setting (lower).

The configuration and volume of the airway were evaluated via image analysis software (Amira 3.1, FEI co., USA, NE).

The airway volumes between the level of the posterior nasal spine and the tip of epiglottis were measured.

\section{Results}

\section{Maximum negative pressure and S_NP}

At the maximal inspiration, the negative pressure within the syringe was $7.8 \mathrm{kPa}$. S_NP was $5.0 \mathrm{kPa}$ (65\% Max.NP).

\section{Evaluation of airway configuration and volume}

The cross-section airway configuration at normal breathing was elliptical shape. On the other hand, the airway under S_NP presented triangle shape (Figure 3).

The airway volumes were $16040 \mathrm{~mm}^{3}$ at normal breathing, and $6567 \mathrm{~mm}^{3}$ under S_NP. The airway volume under S_NP decreased to $41 \%$, compared with normal breathing (Figure 4).

\section{Discussion}

We hypothesized that there is a structural weakness of airway is

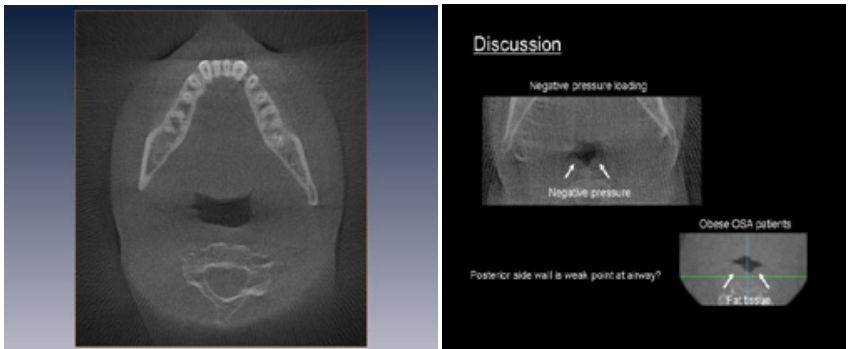

Figure 3: Normal breathing (upper) and Airway configuration under S_NP (lower).

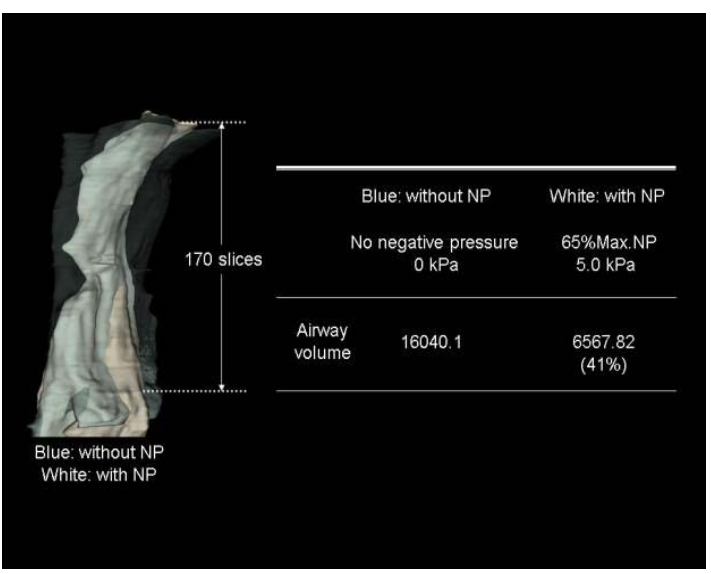

Figure 4: Airway volume under S_NP compared with normal breathing.

in the posterolateral region.

In the volunteer, the airway under S_NP presented triangle shape. This finding provides strong support for our hypothesis.

There is retropharyngeal space (RPS) [2], in the posterolateral region of the airway. The RPS extends from the clivus to the upper mediastinum, lies posterior to the pharynx and esophagus, and is anterior to the prevertebral musculature. It is bounded by the buccopharyngeal fascia anteriorly, the prevertebral fascia posteriorly, and the carotid space laterally. The RPS can be further divided into the suprahyoid and infrahyoid RPS, each with different contents. The suprahyoid RPS contains fat and lymph nodes, whereas the infrahyoid RPS contains only fat.

Obesity is an important risk factor for the development of OSA. Most OSA patients are overweight, and OSA is substantially more common in obese individuals. The exact mechanism by which obesity causes OSA was unclear.

In 2007, we reported the abnormal findings on CBCT images of OSA patients. As one of the morbid findings, there was airway configuration change on the axial slab. The triangular airway form was present in all high BMI patients $\left(26.4-45.3 \mathrm{~kg} / \mathrm{m}^{2}\right)$ [3]. We speculated that increased fat tissue in the neck region may compress the retropalatal and retroglossal airway form causing a triangular shape and decreasing the available space and increasing its collapsibility [4].

In 2007, Ogawa et al [5], observed the airway configuration on the axial slabs of CBCT data. They concluded that the OSA group 
presented a concave or elliptic shaped airway and the non-OSA group presented a concave, round, or square shaped airway.

However, they focused on anterior-posterior and lateral diameters of the airway on axial slab. The criteria of qualitative observation were not certainly defined. In addition, they observed only narrowest cross sectional area. It was a possibility that the triangular shaped airway included in their cases, because one of their figure showed it.

Negative pressure was used to assess the upper airway collapsibility [6]. A previous study investigated the effect of non-rapid-eyemovement sleep on 1) the tensor palatini (TP) electromyogram (EMG) response to rapid-onset negative-pressure generations (NPG) in the upper airway and 2) the collapsibility of the retropalatal airway during these NPGs. They concluded that the brisk reflex response of the TP muscle to negative pressure during wakefulness is markedly reduced during non-rapid-eye-movement sleep, in association with a more collapsible retropalatal airway.

However, there was no research regarding to the alteration of airway for negative pressure.

In this short report, it could observe the airway configuration under the negative pressure. As a result, the airway may be obstructed from posterolateral direction by fat tissue or negative pressure. This short report provided an importance of research regarding to the anatomical weak region in the airway.

\section{References}

1. Shigeta $Y$, Enciso R, Ogawa T, Shintaku WH, Clark GT. Correlation between retroglossal airway size and body mass index in OSA and non-OSA patients using cone beam CT imaging. Sleep Breath. 2008; 12:347-352.

2. Debnam JM, Guha-Thakurta N. Retropharyngeal and Prevertebral Spaces: Anatomic Imaging and Diagnosis. OtolaryngolClin North Am. 2012; 45: 12931310.

3. Shigeta Y, Enciso R, Ogawa T, Shintaku WH, Clark GT. Upper Airway Alterations/Abnormalities in a Case Series of Obstructive Sleep Apnea Patients Identified with Cone-Beam CT. Int J Comput Assist Radiol Surg. 2007;2:402-404.

4. Shelton KE, Woodson $\mathrm{H}$, Gay S, Suratt PM. Pharyngeal fat in obstructive sleep apnea. Am Rev Respir Dis.1993; 148: 462-466.

5. Ogawa T, Enciso R, Shintaku WH, Clark GT. Evaluation of cross-section airway configuration of obstructive sleep apnea. Oral Surg Oral Med Oral Pathol Oral RadiolEndod. 2007; 103:102-108.

6. Wheatley J, Tangel D, Mezzanotte W, et al. Influence of sleep on response to negative airway pressure of tensor palatini muscle and retropalatal airway. J Appl Physiol. 1993; 75: 2117-2124.
Austin J Otolaryngol - Volume 6 Issue 1 - 2019

ISSN : 2473-0645 | www.austinpublishinggroup.com

Shigeta et al. () All rights are reserved
Citation: Hirai S, Ando E, Shigeta Y, Ishikawa C, Ito T, Shigemoto S, et al. Is there Weakness Area in Airway? Austin J Otolaryngol. 2019; 6(1): 1106. 